Journal of Computer Science 6 (8): 872-879, 2010

ISSN 1549-3636

(C) 2010 Science Publications

\title{
Edge Tracing Manipulation of Clothes Based on Different Gripper Types
}

\author{
${ }^{1}$ Khairul Salleh Mohamed Sahari, ${ }^{2}$ Hiroaki Seki, ${ }^{2}$ Yoshitsugu Kamiya and ${ }^{2}$ Masatoshi Hikizu \\ ${ }^{1}$ Department of Mechanical Engineering, University Tenaga National, Kajang, Selangor, Malaysia \\ ${ }^{2}$ School of Mechanical Engineering, Kanazawa University, Kanazawa, Ishikawa, Japan
}

\begin{abstract}
Problem statement: Edge tracing is considered important for deformable object manipulation in order to spread and reveal the original shape of an object before it can be sorted. It is important in unfolding clothes to reveal its original state during sorting. Tracing in this study context involved tracing the clothes edge, with the robot arm movement based on feedback from sensors. Development of special tools for deformable object manipulation is also considered to be important as well and must consider the properties of the deformable objects. Approach: This study introduced three robotic grippers developed to handle clothes for home service robots. For each gripper, a unique tracing algorithm was developed to fully utilize the capability of the gripper in edge tracing. Results: Each developed gripper had been tested and edge tracing algorithms have been developed to suit the gripper's capabilities. Experimental results demonstrated the uniqueness of each gripper and the effectiveness of the tracing algorithm developed. Conclusion: The results showed that the grippers are capable of tracing the edge of clothes and reveal the second corner of the clothes. Inchworm gripper is proven to be the most promising gripper for clothes manipulation.
\end{abstract}

Key words: Roller gripper, inchworm gripper, edge tracing, deformable object, home service robot

\section{INTRODUCTION}

Clothes manipulation by robot is considered one of the challenging tasks because of its deformability. Many researchers are starting to focus more on this task. The research might be theoretical, practical or both (Kita et al., 2004; Wada et al., 2001; Osawa et al., 2006; Saha and Isto, 2006; Moll and Kavraki, 2006; Howard and Bekey, 1999; Schmidt and Henrich, 2001). In clothes handling, the robot must first recognize the shape of clothes. Due to contact and gravity, clothes rarely show their original shape. In this case, edge tracing is important in object recognition. The method is used to spread clothes, which is important in folding or hanging clothes out to dry (Salleh et al., 2008). Spreading clothes basically involves holding two corners of the clothes to spread or fold them (Hamajima and Kakikura, 2000). The problem is finding the appropriate corners. Vision sensors help find corners (Ono et al., 1995), but corners are not necessarily visible or exposed to the camera, causing the robot to hold the clothes unsatisfactorily. Even when a camera detects a corner, the corner may not be appropriate. By using tracing manipulation to find the second corner, it is proven that the second corner found is that next to the initially found corner and not the one apposite it
(Salleh et al., 2008). But there are also problems concerning tracing manipulation. One of the major problems is how to retrieve the fabric when it is in danger of slipping away from the gripper. The robot may be able to detect that the fabric is about to slip but it is hard to retrieve or prevent it. If the robot tries to regrasp the fabric, it would probably slip away. If the robot tries to retrieve the fabric without regrasping it, it would most probably drag the fabric along, flexing it instead of retrieving it. This is due to the fact that deformable objects are sensitive to contact forces. The robot can be programmed to retrace the fabric from the beginning but this will take time. The solution to this problem is to design a special tool that can trace the edge smoothly and can perform fabric retrieving. This study presents three different type of grippers used to handle clothes, the first one being the basic standard gripper for the purpose of benchmarking. Ability to perform the retrieving process is incorporated into the other two grippers. The developed grippers are evaluated in terms of their efficiency and also effectiveness.

\section{MATERIALS AND METHODS}

Clothes manipulation system: Figure 1 shows the clothes manipulation system. In this research, one Js2

Corresponding Author: Khairul Salleh Mohamed Sahari, Department of Mechanical Engineering, University Tenaga National, Kajang, Selangor, Malaysia 
(manufacturer: Kawasaki Heavy Industries) with a 6 Degree Of Freedom (DOF) and one RCH-40 (Yamaha) with a 5 DOF are used. Both are articulated robotic arm and are equipped with customized robot grippers designed for clothes manipulation. A vision sensor (Tokyo Denshi Kogyo CCD camera with a $6 \mathrm{~mm}$ focus range and a focal ratio of $1: 1.4)$ is located in a fixed position in front of the two robots. The images taken by the CCD camera are in 8 bit grayscale format and $640 \times 480$ pixels in size. Two personal computers are used in this research. A Dual-RAM board is used for data accessing and transferring between the two computers. An image processing board TRV-CPW5 (Fujitsu) that has image tracking function is attached to the main computer to speed up image processing. The control unit for RCH-40 is also connected to the main computer through RS-232C port. The sub computer houses all the other data acquisition cards. The sub computer also controls the Js2 using the RS-232C port. Basically, the main computer runs the main program and will use timer interrupts to give instructions to the sub computer.

Robotic grippers: To study the importance of using appropriate tools in clothes manipulation, three types of robotic grippers were developed and tested. These grippers are attached to the Js2 robot since Js2 is used to trace the edge of the clothes. The grippers are shown in Fig. 2. The basic properties of the grippers are shown in Table 1.

Basic gripper: Basic gripper in this study context is an open-close gripper controlled by a DC servo motor equipped with rotary encoder from Maxon motors $(18 \mathrm{~V}$, $3.0 \mathrm{~W}$, lift-torque $10.8 \mathrm{mNm}$, max rpm $16000 \mathrm{rpm}$ ) via spur gears and two-way ball screw with a $1 \mathrm{~mm}$ lead. The encoder's resolution is 100 pulse/rotation. The unique part about the gripper compared to other grippers in the market is the thin part on both fingers that are sensitive to changes in force. Strain gages are attached on both sides of the thin part of a finger where the strain value is determined from the whetstone bridge through a strain amplifier. When the gripper closes, the fingers will push each other backward and the average strain from two fingers is used to calculate the force applied.

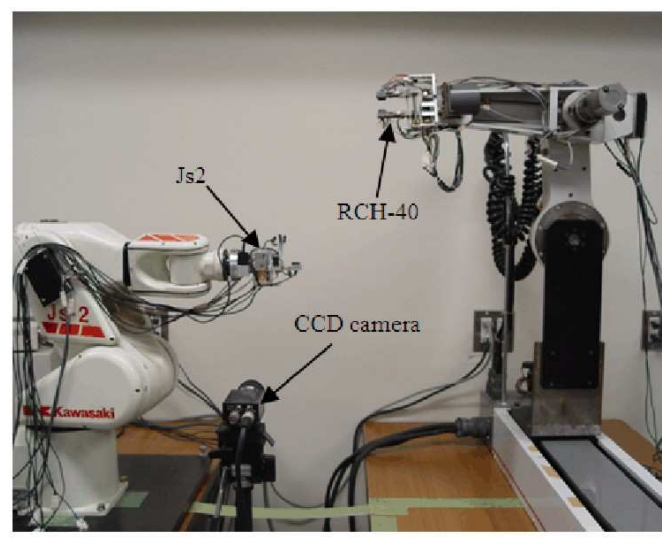

Fig. 1: Clothes manipulation system

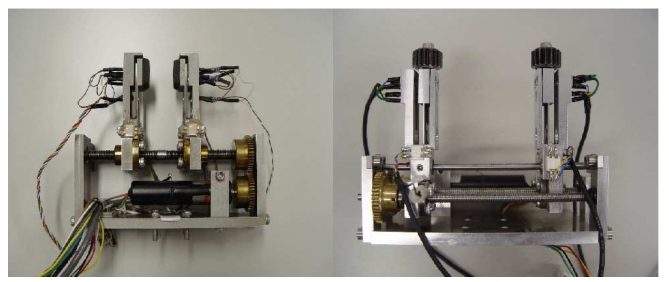

(a)

(b)

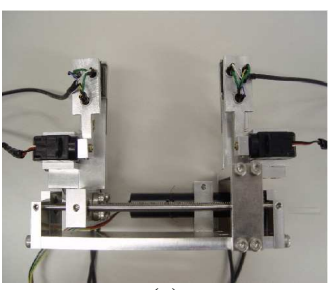

(c)

Fig. 2: Robotic grippers (a) Basic gripper; (b) Roller gripper; (c) Inchworm gripper

Table 1: Gripper basic properties

\begin{tabular}{|c|c|c|c|}
\hline Gripper specification & Basic gripper & Roller gripper & Inchworm gripper \\
\hline Dimension & $107 \times 90 \times 40 \mathrm{~mm}$ & $107 \times 80.1 \times 56.75 \mathrm{~mm}$ & $130 \times 96.1 \times 56.75 \mathrm{~mm}$ \\
\hline Maximum opening (mm) & 35 & 45 & 15 \\
\hline Number of infrared sensors & 4 & 3 & 6 \\
\hline Number of strain gages Sensors & 4 & 4 & 4 \\
\hline Thickness of thin part (mm) & 0.7 & 0.4 & 0.5 \\
\hline $\begin{array}{l}\text { Maximum allowable grasping } \\
\text { force }(\mathrm{gf})\end{array}$ & 558 & 95.1 & 339.5 \\
\hline Actuator used & $\begin{array}{l}\text { Maxon }(18 \mathrm{~V}, 3.0 \mathrm{~W} \text {, lift torque } \\
10.8 \mathrm{mNm}, \max \mathrm{rpm} 16000 \mathrm{rpm})\end{array}$ & $\begin{array}{l}\text { Maxon }(18 \mathrm{~V}, 3.0 \mathrm{~W} \text {, lift torque } \\
10.8 \mathrm{mNm}, \max \mathrm{rpm} 16000 \mathrm{rpm})\end{array}$ & $\begin{array}{l}\text { Maxon }(18 \mathrm{~V}, 3.0 \mathrm{~W} \text {, lift torque } \\
10.8 \mathrm{mNm} \text {, max rpm } 16000 \mathrm{rpm}) \\
\text { Futaba MS-1 (x2) }\end{array}$ \\
\hline
\end{tabular}




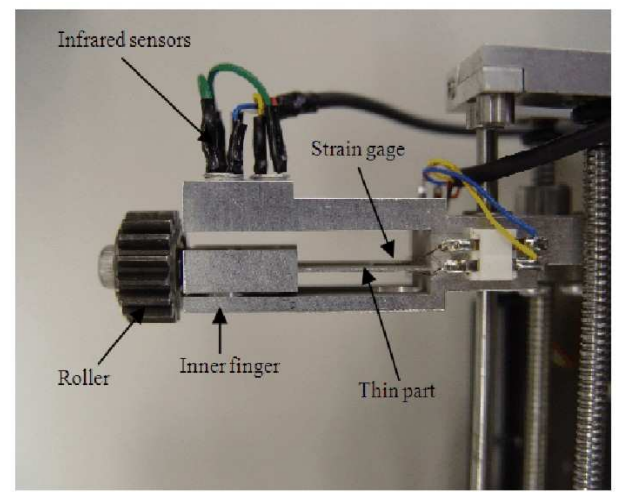

Fig. 3: Roller gripper in focus

This is useful in maintaining the grasping force during tracing. A thick part just behind the thin part acts as a stopper in case of excessive force to prevent the thin part from failure. 4 sets of infrared sensor (Toshiba) are embedded onto the fingers to give information on whether clothes or fabric is inside the gripper or not. Based on the task, the thin part is designed to withstand $558 \mathrm{gf}$ of force before it touches the thick part behind. This is more than enough for clothes handling. $\mathrm{RCH}-40$ is always equipped with a basic gripper.

Roller gripper: Roller gripper is a customized version of the basic gripper. Basically, this gripper is the same as the normal gripper except for the roller fingertips and the inner fingers. The gripper also has 3 sets of infrared sensors compared to 4 sets for the basic gripper. From numerous clothes manipulation experiments, it was found that 3 sets of infrared sensors are sufficient for clothes manipulation. The rollers at fingertips are made of identical spur gears (diameter: $14 \mathrm{~mm}$, number of teeth: 14 , module: 0.875 ) attached to the fingertips via roller bearings and can freely rotate on their axes. The purpose of the roller tips is to enable smooth movement of the gripper along the clothes edge when the tracing direction is parallel with the rollers' rotation axis. The gripper is also more sensitive to force change. This is due to the thickness of the thin part being $0.4 \mathrm{~mm}$ compared to $0.7 \mathrm{~mm}$ for the basic gripper. Similar to the basic gripper, strain gages are used to measure the grasping force. The maximum applicable load before the thin part touches the stopper is $95.1 \mathrm{gf}$. The gripper also has inner fingers used to firmly grasp the clothes Fig. 3. This is because the clothes might slip when grasped using the rollers alone since they have no brakes. When a big enough force is applied to the gripper, the thin part will bend outwards and enables the inner fingers to grasp the clothes. This depends on the thickness of the clothes. The gripper opening is controlled using the same model as the basic gripper via 2 spur gears with a 1:1.6 gear ratio and two ball screws with a $1 \mathrm{~mm}$ lead. The maximum opening is $45 \mathrm{~mm}$.

Inchworm gripper: Unlike the basic gripper or the roller gripper, the inchworm gripper has two sets of grippers, one fixed (fixed gripper) and one slide-able (slide gripper) and 3 actuators, one for moving the slide gripper sideways and the other two for opening and closing of the grippers. The sliding motion is controlled by the same Maxon motor used in the other two grippers, the power distributed through a set of spur gears with a $1: 1$ gear ratio to a ball screw with a $1 \mathrm{~mm}$ lead. The opening and closing of the grippers are controlled by micro analog servos MS- 1 from Futaba $(4.8 \mathrm{~V}$, rotation speed $0.002 \mathrm{sec} / \mathrm{deg}$ (at $4.8 \mathrm{~V}$ ), torque $1.2 \mathrm{Kg} . \mathrm{cm}($ at $4.8 \mathrm{~V}))$. Unlike the other grippers, the actuators only control one of the two fingers of a gripper. The servos are directly connected to the fingers which makes the opening and closing motion to be rotational. This means the bigger the opening the harder for the infrared sensors to function properly because the opening is in rotation form and not linear. The maximum finger rotations for slide gripper $(\psi 1)$ and fixed gripper $(\psi 2)$ where the infrared sensors can function properly are approximately $20^{\circ}\left(0^{\circ}\right.$ is when the two fingers barely touches each other). There are 3 sets of infrared sensors on each gripper. The layout of the infrared sensors is the same as the roller gripper. The slide gripper can slide a maximum $60 \mathrm{~mm}$ away from the fixed gripper. Strain gages are attached to the thin part of the fixed finger on both the slide and fixed grippers. The thin parts of the grippers are $0.5 \mathrm{~mm}$ in thickness and should bear up to $339.5 \mathrm{gf}$ of force before touching the thick parts that act as stoppers. This inchworm gripper will enable the clothes to be firmly grasped at all times during clothes manipulation.

Edge tracing in finding the second corner: In this research, after the robot has found and successfully grasped the first corner, it will search for the corner next to the first corner in order to spread the towel correctly. Although the usage of vision sensors plays a big role in helping to find a corner (Ono et al., 1995), the corners of the clothes are not necessarily visible or exposed to the camera. Even if the camera can detect a corner, it doesn't mean that the corner is the right one. The robot may end up not holding a true corner of the clothes or holding a wrong corner thus resulting in improper spreading of the clothes. The method for finding the first corner can be used here but most probably the corner found would not be a corner next to the one being grasped. Edge tracing method in the other hand would not just enable the discovery of the second 
corner but also ensures that the corner is the one next to the first corner (Salleh et al., 2008). Other merits of using this method are the process can be performed in the air, so a working platform is not necessary (Salleh et al., 2010), besides being robust to most shapes and sizes. Before the edge tracing manipulation can take place, the tracing gripper (Js2) must be holding the edge of the clothes just beneath the first corner.

Edge tracing using basic gripper: The algorithm developed for the basic gripper is the basis for all edge tracing algorithms. The idea is to apply a certain amount of force while the gripper traces the edge of the clothes based on infrared sensors feedback. From time to time, vision sensor will check the progress, whether the next corner has been found or not. Certain infrared sensors feedback patterns also tell that possible corner has been reached. The flow of the tracing manipulation is shown in Fig. 4.

Edge tracing using roller gripper: There is one major problem during edge tracing using basic gripper. If the gripper accidently traced too near the edge and is about to release the towel, the feedback from the infrared sensors can detect this. But due to the structure of the gripper and the nature of the clothes, it is very difficult to prevent the clothes from falling. Since the whole process of clothes spreading is done on the air, the weight of the clothes will work downwards, away from the gripper. There is also the factor of friction between the gripper and the clothes. To reduce the contact force between the gripper and the clothes, a roller mechanism is designed at the fingertip of each finger. This will reduce the friction between the clothes and the gripper.

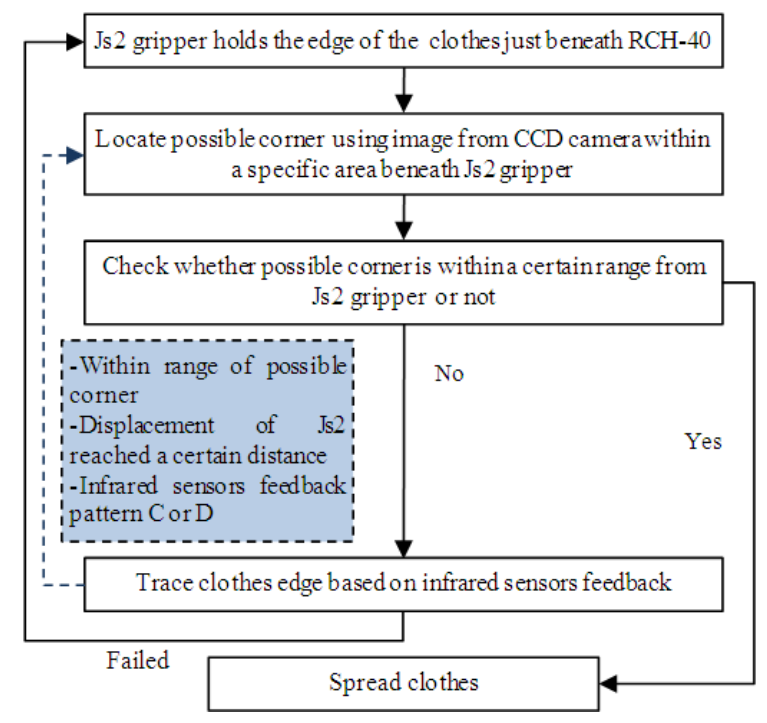

Fig. 4: Tracing flowchart for basic gripper
This then enables the retrieving movement (movement backwards) of the gripper and increases the flexibility of the tracing process. The flow of the tracing manipulation with the retrieving process is shown in Fig. 5. The tracing algorithm for basic gripper is used as the basis behind the development of tracing algorithm for roller gripper.

Edge tracing using inchworm gripper: Although the roller gripper can increase the flexibility of the tracing in particular and clothes handling in general, there are still risks of the clothes slipping away from the gripper. In order to overcome this issue, the inchworm gripper is developed. The main reason behind designing and developing the inchworm type grippers is to enable the edge of the towel to be firmly grasped at all times during edge tracing. This will definitely increases the success rate of the tracing manipulation process. Having two pairs of grippers on one robot hand certainly helps this cause. The tracing manipulation algorithm using the inchworm gripper is shown in Fig. 6. The basic idea is to have two grippers inside the robot hand with the gap between the two grippers adjustable. The movement pattern of an inchworm is applied into the tracing algorithm. While one gripper traces along the edge, the other gripper will firmly holds the towel. The grippers will alternately trace the edge until a second corner is found. The merit of using this technique is precise force control during tracing will become unnecessary.

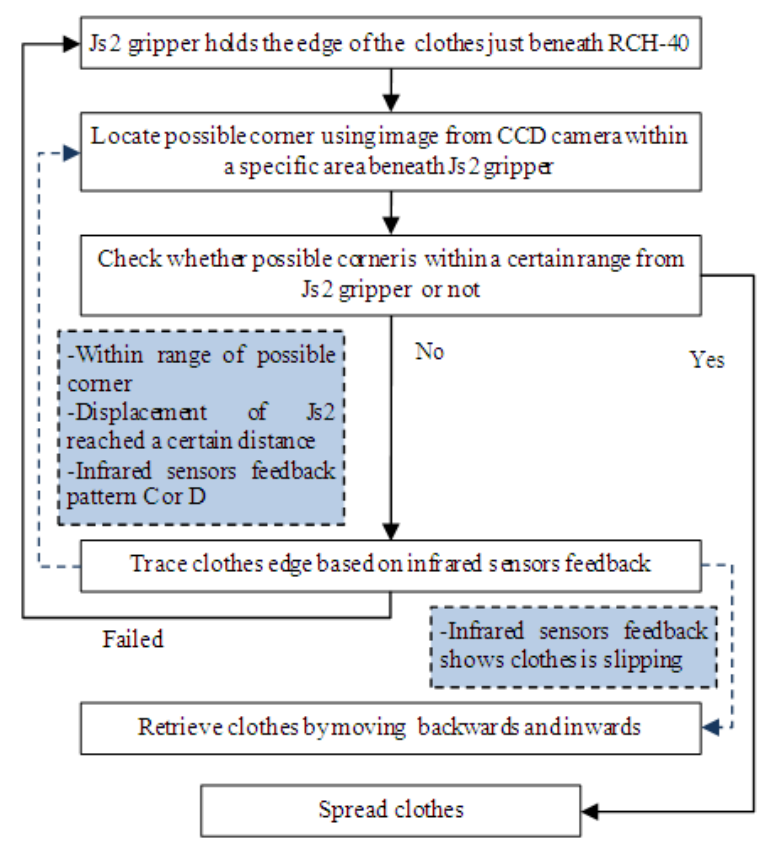

Fig. 5: Tracing flowchart for roller gripper 
During tracing manipulation using previous grippers, force control plays a very important factor. Applying too much force will result in the gripper dragging the fabric instead of tracing it. But if too little force is applied the fabric will slip away. But by using the inchworm type grippers, precise force will become unnecessary due to the fact that at least one of the grippers will be holding the fabric firmly at one time. Omitting the force control will also reduce the failure rate.

Corner confirmation using vision sensor: Regardless of the gripper used, corner confirmation process will be based on two criteria. First, it is based on the feedback of the infrared sensors. Patterns C and D indicate that a possible corner is reached. Corner confirmation process will also take place after Js2 has traced a predetermined distance, S. An image close to the Js2 gripper tip is captured. If a corner is reached or the corner is close enough to the Js2 gripper tip ( $2 \mathrm{~cm}$ or lesser), the tracing process will stop and the robot is going to spread the clothes.

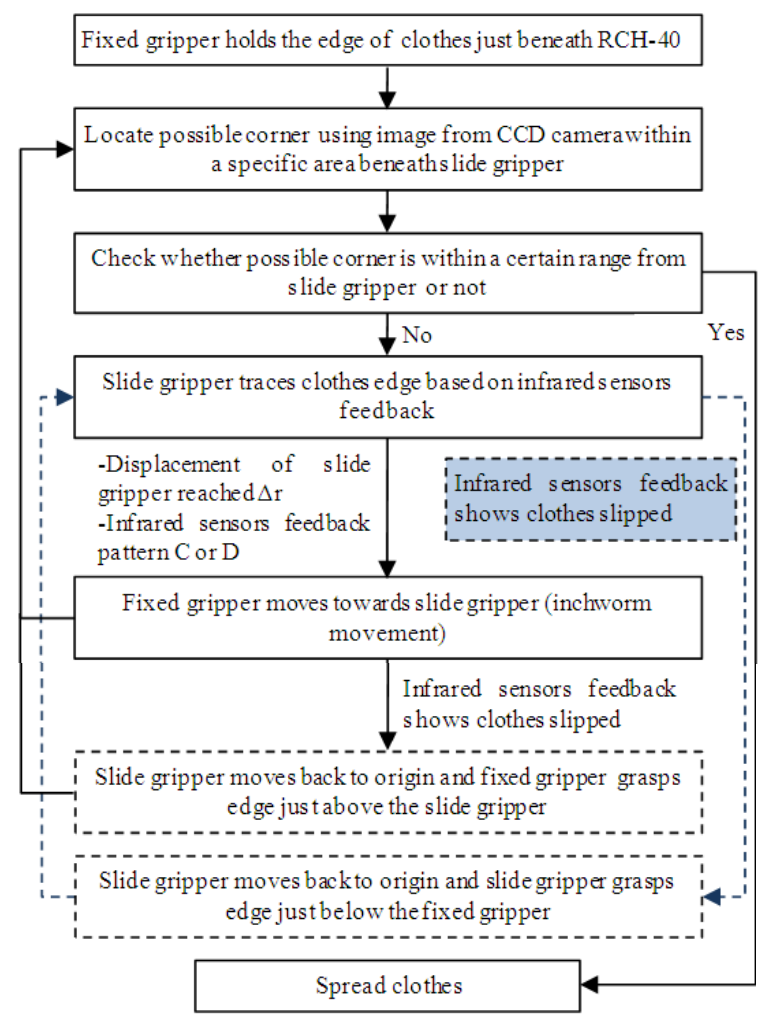

Fig. 6: Tracing flowchart for inchworm gripper

\section{RESULTS}

Tracing experiments were conducted to evaluate the algorithms and the grippers. Rectangular shaped towel (properties: color orange, size $280 \times 280 \mathrm{~mm}$, thickness $2.28 \mathrm{~mm}$, mass per unit area $3.7 \mathrm{~g} \mathrm{~mm}^{-2}$, coefficient of friction $\mu 0.615$, stretch rate $0.005 \mathrm{~mm}$ $\mathrm{gf}^{-1}$ ) is used in all experiments for simplicity since the side-by-side corners are all connected in a straight line. All experiments were started with the Rch- 40 gripper holding a corner of the towel. The range for image processing during corner confirmations is set at $80 \times 110$ pixels with the right topmost point 10 pixels below the end of Js2 gripper on the screen. One pixel corresponds to $0.94 \mathrm{~mm}$ of length at a distance of $0.35 \mathrm{~m}$ in front of the camera. Real time control is applied to Js2 robot during tracing where the tracing velocity is set at 0.02 $\mathrm{m} \mathrm{sec}^{-1}$. Experiments were conducted 30 times each.

Experiments using basic gripper: Figure 7 shows example data obtained from a successful tracing experiment. From Fig. 7a, it can be observed that Js2 only moves forward throughout the tracing process. The positions of possible corners are also indicated.

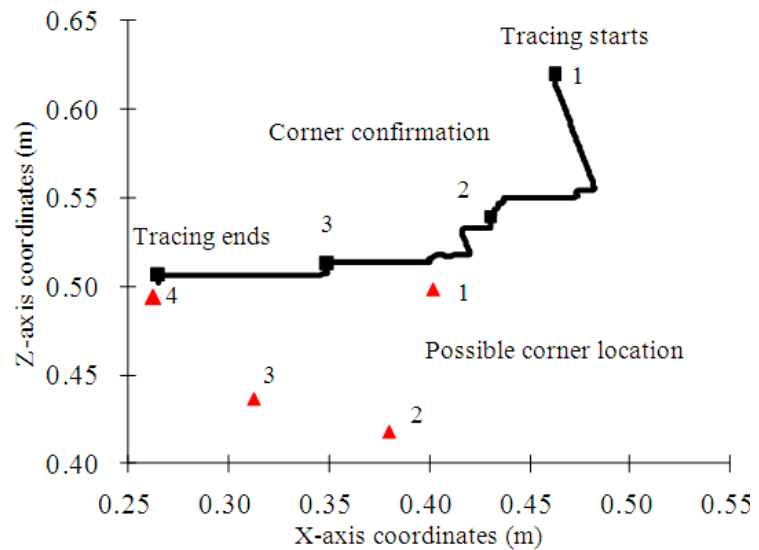

(a)

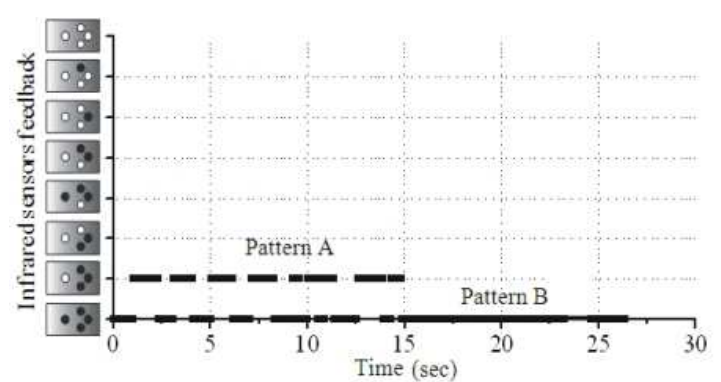

(b)

Fig. 7: Sample data from tracing experiment-basic gripper (a) Js2 tracing trajectory; (b) Js2 infrared sensors feedback 
It can be observed that the trajectory path did not go near the position of the possible corners at times. This is due to the gripper tracing too deep into the clothes. Feedback from infrared sensors on the Js2 gripper is shown in Fig. 7b. From the feedback, it can be said that the Js2 gripper traced in almost zigzag pattern and in most cases, the feedback is between patterns A and B.

Experiments using roller gripper: Figure 8 shows example data obtained from a successful tracing experiment. From Fig. 8a, it can be observed that Js2 did not only move forward, but at times it moved backwards (retrieving process) as well during the tracing process. Feedback from infrared sensors on the Js2 gripper is shown in Fig. 8b. From the feedback, it can be said that the gripper traced near or along the edge of the towel. Figure 8c shows the images taken by the vision sensor during corner confirmation.

Experiments using inchworm gripper: Figure 9 shows example data obtained from a successful tracing experiment using inchworm gripper. Figure 9a shows the trajectory of Js2 during tracing. It can be observed that the Js2 trajectory is not as complicated as the previous two grippers. Feedback from infrared sensors on the slide and fixed gripper are shown in Fig. 9b and $\mathrm{c}$ respectively. From the feedback, it can be said that the gripper traced near or along the edge of the towel. Figure 10 shows the snapshots taken during edge tracing in which the inchworm movement can be observed.

Overall efficiency and tracing time: The average efficiency and the time taken for the tracing process for basic, roller and inchworm grippers are listed in Table 2. Based on the percentage, the success rates for roller and inchworm grippers are higher than the basic gripper. In terms of time consumption, roller gripper is the fastest but the complexity of the algorithm is not the same for all 3 grippers. The average required time is influenced by the simplicity of the algorithm as well as the ability of the robot to find the corner on its first try. Of course, the time can be further reduced through optimization of parameters as well as the improvement of the current algorithm. Average time is not a major concern for this study. Based on previous experiments and also observation, faster tracing speed will usually increase failure rate. But again, since all failures can be detected, the processes can always be repeated until the robot succeeds in spreading the clothes which is very important.

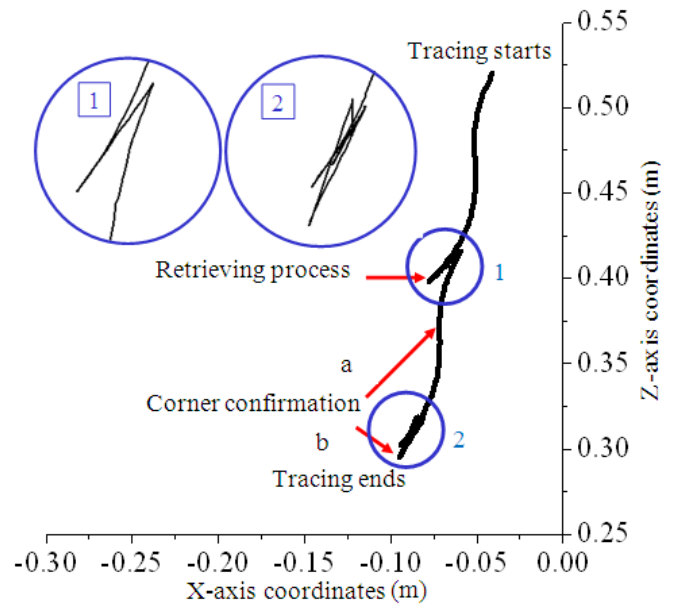

(a)

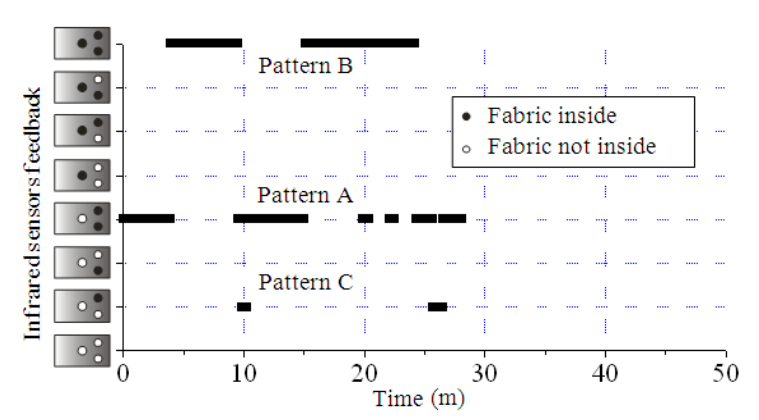

(b)

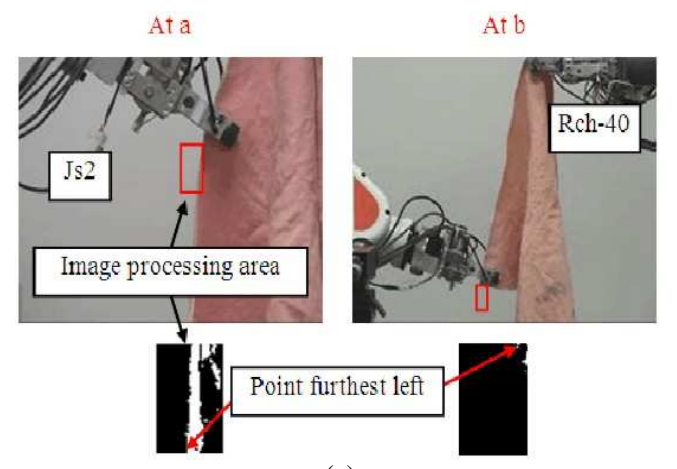

(c)

Fig. 8: Sample data from tracing experiment-roller gripper (a) Js2 tracing trajectory; (b) Js2 infrared sensors feedback; (c) corner confirmation process

Table 2: Tracing results

\begin{tabular}{llll}
\hline Gripper specification & $\begin{array}{l}\text { Basic } \\
\text { gripper }\end{array}$ & $\begin{array}{l}\text { Roller } \\
\text { gripper }\end{array}$ & $\begin{array}{l}\text { Inchworm } \\
\text { gripper }\end{array}$ \\
\hline $\begin{array}{l}\text { Success rate (\%) } \\
\begin{array}{l}\text { Average time (sec) } \\
\text { Successful 1st attempt) }\end{array}\end{array}$ & 60 & 73.3 & 83.3 \\
$\begin{array}{l}\text { Average time (sec) } \\
\text { (until successful) }\end{array}$ & 62 & 31.0 & 54.0 \\
\hline
\end{tabular}




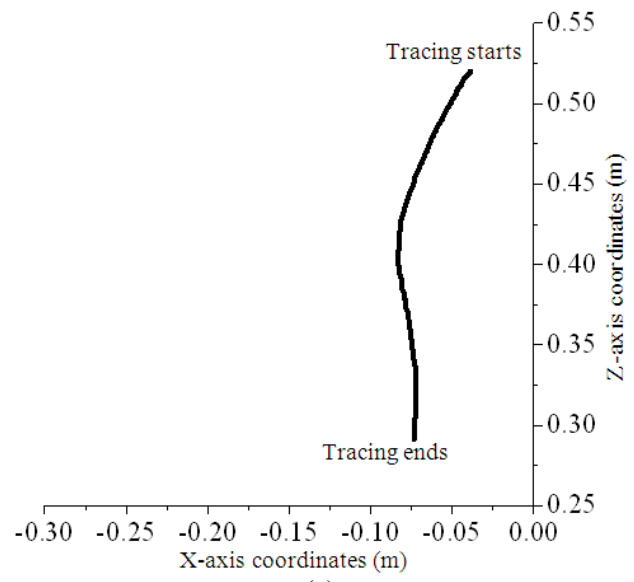

(a)

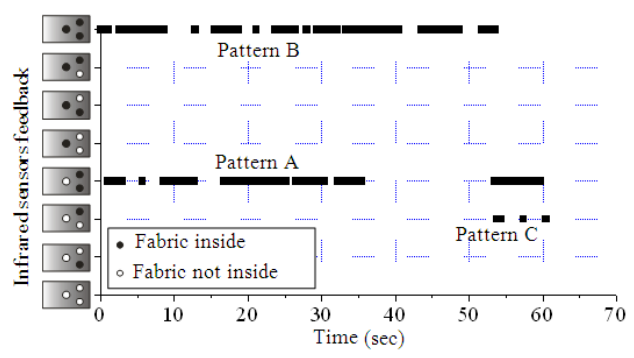

(b)

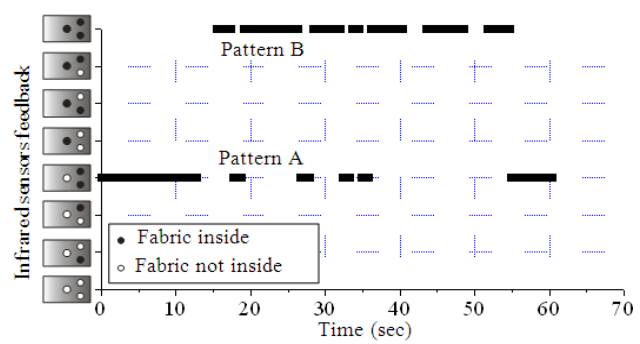

(c)

Fig. 9: Sample data from tracing experiment-inchworm gripper (a) Js2 tracing trajectory; (b) Slide gripper infrared sensors feedback; (c) Fixed gripper infrared sensors feedback

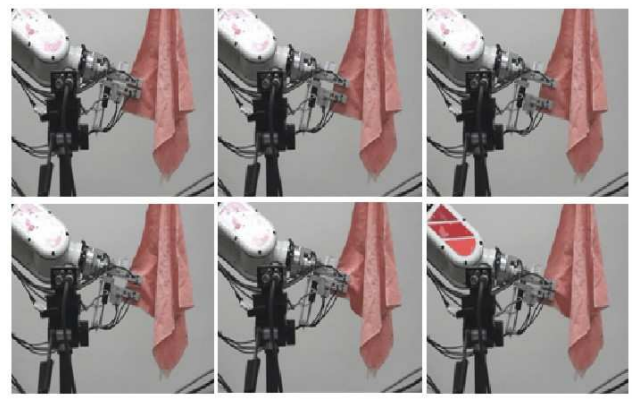

Fig. 10: Scenes during tracing-inchworm

\section{DISCUSSION}

The experimental results showed that the inchworm gripper gives the best result. This is basically due to its two sets of grippers in parallel. This provides the easiest control algorithm for tracing manipulation. The other grippers require adequate force control to maintain grasping and in the same time be able to further trace the edge. Inchworm gripper on the other hand does not require any precise force control. At all times during tracing, at least one of the two set of grippers will be firmly holding the clothes. Since the maximum sliding distance of the slide gripper is $60 \mathrm{~mm}$, the slide gripper has managed to trace the edge without having to properly grasp the edge. This is because although clothes can deform easily, the deformation at a point close to the point being grasped is usually very small. By using this concept, tracing can be done with much ease and success. In terms of speed, the time taken for inchworm gripper is longer for the time being. Optimization of the parameters such as the speed of the DC motor and the servo motor will definitely speed up the tracing process.

\section{CONCLUSION}

A study on edge tracing by three different grippers for clothes manipulation is presented. The study discussed the difference between the three grippers on the mechanism and also the algorithm for clothes manipulation. Having the basic gripper as the benchmark, roller gripper is better than basic gripper due to the presence of the rollers on the fingertips. Retrieving process can be easily done which increases the flexibility of the tracing process as well as increasing the success rate. Inchworm gripper on the other hand doesn't require any force control during tracing as two sets of grippers side-by-side are always close to each other and the risk of the clothes slipping is very small compared to basic and roller grippers. In terms of practicality, inchworm gripper may be useful for a lot of other applications compared to the roller gripper. In terms of simplicity, roller gripper requires lesser actuators. Hence, it is less complicated. It can be concluded that depending on the gripper, the manipulation of deformable object can be executed but with different algorithms, speed and reliability. In order to ease the process, inchworm gripper provides the best solution in terms of simplicity and reliability.

There are still a lot of research elements available such as to further improve the method, to optimize and also to apply the current method to other clothes. 


\section{ACKNOWLEDGEMENT}

This research is supported by the Ministry of Higher Education of Malaysia under the project code: FRGS/ FASA1-2009/ TEKNOLOGI and KEJURUTERAAN/ UNITEN/ 9 and also by research grant from Kanazawa University, Japan.

\section{REFERENCES}

Hamajima, K. and M. Kakikura, 2000. Planning strategy for task of unfolding clothes. Robot. Autonom. Syst., 32: 145-152. DOI: 10.1016/S0921-8890(99)00115-3

Howard, A.M. and G.A. Bekey, 1999. Intelligent learning for deformable object manipulation. Proceeding of the IEEE International Symposium on Computational Intelligence in Robotics and Automation, Nov. 8-9, IEEE Xplore Press, Monterey, Canada, pp: 15-20. DOI: 10.1109/CIRA.1999.809935

Kita, Y., F. Saito and N. Kita, 2004. A deformable model driven visual method for handling clothes. Proceeding of International Conference on Robotics and Automation, Apr. 26-May 1, IEEE Xplore Press, New Orleans, USA., pp: 3889-3895. DOI: 10.1109/ROBOT.2004.1308874

Moll, M. and L.E. Kavraki, 2006. Path planning for deformable linear objects. IEEE Trans. Robot., 22: 625-636. DOI: 10.1109/TRO.2006.878933

Ono, E., N. Kita and S. Sakane, 1995. Strategy for unfolding a fabric piece by cooperative sensing of touch and vision. Proceeding of IEEE/RSJ International Conference on Intelligent Robots and Systems Human Robot, Aug. 5-9, IEEE Xplore Press, Pittsburgh, USA., pp: 441-445. DOI: 10.1109/IROS.1995.525922
Osawa, F., H. Seki and Y. Kamiya, 2006. Clothes folding task by tool-using robot. J. Robot. Mechatron., 18: 618-625.

Saha, M. and P. Isto, 2006. Motion planning for robotic manipulation of deformable linear objects. Proceeding of the IEEE International Conference Robotics and Automation, May 15-19, IEEE Xplore Press, Orlando, USA., pp: 2478-2484. DOI: 10.1109/ROBOT.2006.1642074

Salleh, K., H. Seki, Y. Kamiya and M. Hikizu, 2008. Inchworm robot grippers for clothes manipulation. Artif. Life Robot., 12: 142-147. DOI: 10.1007/s10015-007-0456-6

Salleh, K., H. Seki, Y. Kamiya and M. Hikizu, 2010. Clothes manipulation by robot grippers with roller fingertips. Adv. Robot., 24: 139-158. DOI: 10.1163/016918609X12586175245158

Schmidt, T.W. and D. Henrich, 2001. Manipulating deformable linear objects: robot motions in single and multiple contact points. Proceedings of the IEEE International Symposium on Assembly and Task Planning, May 28-29, IEEE Xplore Press, Fukuoka, Japan, pp: 435-441. DOI: 10.1109/ISATP.2001.929073

Wada, T., B.J. McCarragher, H. Wakamatsu and S. Hirai, 2001. Modeling of shape bifurcation phenomena in manipulations of deformable string objects. Adv. Robot., 15: 833-846. DOI: $10.1163 / 156855301317198151$ 